International Review of Research in Open and Distributed Learning Volume 21, Number 2

April - 2020

\title{
An Exploration Into the Importance of a Sense of Belonging for Online Learners
}

Susi Peacock ${ }^{1}$, John Cowan ${ }^{2}$, Lindesay Irvine ${ }^{3}$, and Jane Williams ${ }^{3}$

${ }^{1}$ University of the West of England, ${ }^{2}$ Edinburgh, Napier University, ${ }^{3}$ Queen Margaret University

\begin{abstract}
Fostering a sense of belonging and a personal connection is seen as fundamental by many educational researchers, regardless of the learning environment. Online learning certainly provides flexible learning opportunities but comes with notable issues. For online learners, nurturing a sense of belonging may present a way of improving their experiences and attainment, as well as reducing attrition rates. Research specifically exploring sense of belonging and online learning is limited. This article addresses that gap and reports on a small-scale exploratory study using qualitative data-collection and analysis methods to investigate the importance, or not, of sense of belonging for postgraduates' online education by exploring the origins and nature of their lived experience of online learning and their sense of belonging therein. Our initial findings emphasise its importance for them as online learners and have identified three significant themes: interaction/ engagement, the culture of the learning, and support. These early findings highlight the importance of these three themes in promoting a sense of belonging and in ensuring that there are opportunities for meaningful group and peer interactions; they will be of interest to all engaged in online education.
\end{abstract}

Keywords: sense of belonging, online learning, online group work, online discussions, interaction, tutoring 


\section{Introduction}

Over the last two decades, there has been a continuing interest in online learning, with numbers of online courses expected to grow further as institutions reach out to more diverse markets (Allen \& Seaman, 2013). Nowadays, it is common for students, especially those studying online, to be employed in a profession but seeking further development, possibly for accreditation. Consequently, the authors refer herein to learners rather than students, aside from when quoting from cited sources, questionnaire returns, or interview transcripts.

Online learning can provide a flexible learning space, allowing learners access to educational opportunities while continuing to fulfil their various professional and familial responsibilities (O’Shea, Stone, \& Delahunty, 2015). By studying in this nurturing environment with like-minded peers, learners can broaden and deepen the skills and abilities required in their chosen career pathways. They gain instant access to upto-date resources and to experts in their subject specialism (Laurillard, 2012). However, for many learners, the online space may be alien, even threatening (Thomas, 2012), resulting in high attrition rates and lower than expected learner attainment compared with campus-based learners (Carr, 2000). This is particularly so when learners must post contributions in online discussions and engage in online group work (Whittaker, 2015). Online learners often report feelings of being "out of their depth," as well as being underconfident in their academic skills and abilities (Baxter, 2012).

In face-to-face campus-based programmes, learners with a strong sense of belonging report feelings of being comfortable in their learning environs and engaged with their studies. Additionally, they consider that their social and cultural values are aligned with those of their university. Such learners are usually more motivated and have a firm belief that they can achieve and will achieve (Matheson \&Sutcliffe, 2018; Meehan \& Howells, 2018). J arvis (2009) and Illeris (2014) both identify that how the learner mobilises the mental energy for learning through motivation, emotion, and volition is critical in driving the character and durability of the learning. Thus, the absence of a sense of belonging for these learners may lead to feelings of anxiety, frustration, and boredom, impacting negatively on their academic life and performance. This is potentially the case in settings where their values and needs seem unappreciated.

Many issues that a sense of belonging seeks to address are also applicable to online learners. With the requirement for learners to cope effectively with a wealth of materials that focus more on the content and on acquisition of knowledge rather than offering incentives, some can find online learning to be a lonely experience from the outset (Illeris, 2014). However, little is known about a sense of belonging on the part of online learners who are engaging in collaborative, community-based learning, and even less is known about how institutions and tutors can and do promote it.

This article reports on exploratory work investigating the origins, nature, and importance —or not—of a sense of belonging in the lived experience of online learning for certain postgraduate online learners. This work was undertaken to extend the limited research into these aspects of collaborative online learning. The chosen methodology enabled action-researching university tutors to obtain illuminative insights into aspects of the learning experiences they were creating and to identify findings that have already been of interest to managers and tutors in the sector. 
In what follows, we use tutor to describe a staff member appointed to support the creative planning of a course before learning activities commence and to facilitate learning during the course (Peacock \& Cowan, 2017). We believe it highly desirable in any online programme for learners to develop a sense of belonging about their experience; and we agree with Thomas, Herbert, and Teras (2014) that strategies that foster online learners' sense of belonging could enhance their educational experiences and improve retention. So we are seeking here to broaden and deepen understandings about sense of belonging in online learning while providing guidance for tutors, professional staff, and institutions about promoting it in online learning. This article and the action research it describes were founded on the theory and findings reported by Thomas et al. (2012). The outcomes will be of interest to tutors working in the online learning space, professional staff such as librarians, support staff in immediate contact with online learners, and senior management and policy makers as they seek to address online learners' needs as identified above.

\section{The Need for Belonging}

\section{Defining the Concept}

The need and desire to feel in some way connected with and related to others, and to be positively regarded by them, is a universal human characteristic, influencing behaviours and perceptions (Baumeister \& Leary, 1995). Individual needs for a sense of belonging vary but are particularly acute in times of flux, stress, and transition (Strayhorn, 2012), as when studying in an unfamiliar environment like online education. The term itself-sense of belonging-seems deceptively clear and explicit; but while many descriptions of it exist, there is a general lack of clarity and consistency among them. Sense of belonging seems to have both psychological and social dimensions for learners, with two defining attributes (Hagerty, Williams, Coyne, \& Early, 1996). The first involves feelings of being accepted, needed, respected, mattering, and valued in a class. The second pertains to feelings of fitting in - being connected with a group, class, department, subject, institution, or all of these (Hausmann, Ye, Schofield, \& Woods, 2007; Vaccaro, Daly-Cano, \& Newman, 2015).

We subscribe to the most frequently cited and accessible educational definition of sense of belonging, due to its emphasis on feelings. It was proposed by Goodenow in 1993, where she describes sense of belonging as comprising feelings of

being accepted, valued, included, and encouraged by others (teachers and peers) in the academic classroom and of feeling oneself to be an important part of the life and activity of the class. More than simple perceived liking or warmth, it also involves support and respect for personal autonomy and for the student as an individual. (Goodenow, 1993, p. 25)

\section{Belonging in Campus-Based Learning}

It has been widely confirmed that campus-based learners value having a sense of belonging, with feelings of being cared and mattering to one or more people in a group who in turn matter to them, although perhaps for different reasons (Strayhorn, 2012). Generally, the concept is linked to Hurtado and Carter's work in 1997, which offers a holistic approach to learner withdrawal, suggesting that learner persistence is a joint 
responsibility of learner and institution. Since then, educational researchers have linked a sense of belonging with improved learner attainment, increased learner satisfaction, and persistence (Hausmann et al., 2007; Locks, Hurtado, Bowman, \&Oseguera, 2008; Vaccaro et al., 2015). Hoffman, Richmond, Morrow, and Salomone (2002-2003) report that "the greater a student's sense of belonging to the university, the greater is his or her commitment to that institution ... and the more likely it is that he or she will remain" (p. 228). Significant work in the United States reports the particular importance of a sense of belonging for learners who perceive themselves to be marginal to campus life and non-traditional according to class, race, ethnicity, sexual identity, income, or disability (Hausmann et al., 2007; J ohnson et al., 2007; Locks et al., 2008; Vaccaro \& Newman, 2017). Strayhorn (2012) deduces that if a sense of belonging is not developed, learners' ability to attend to the task at hand (studying and fulfilling the goals of higher education) will be impeded. The lead author of an extensive research project involving 22 higher educational institutions in the United Kingdom concludes that "at the heart of successful retention and success is a strong sense of belonging in [higher education] for all students" (Thomas, 2012, p. 6). Thus, the concept is recognised and valued for campus-based learning.

\section{Belonging in Online Learning}

The need for belonging is one of the most important needs for all students to function well in all types of learning environments [emphasis added]. (J ackson et al., 2010, para. 5)

Some research has been published regarding sense of belonging in online learning. Garrison (2011) articulates the importance of belonging in online communities of inquiry, stating that we should "establish a feeling of belonging to the critical community that must develop over time" (p. 32). The authors (Peacock \& Cowan, 2019) have used an adapted version of the community inquiry framework to frame specific suggestions for action to nurture online learners' sense of belonging. Thomas et al. (2014) offer similar insights into the tutor and learner perspectives of online learning and an associated sense of belonging, emphasising its importance and that engagement and collaboration with peers fosters "a sense of camaraderie that diffuse[s] some of the isolation" (p.76) associated with online learning. Such interactions reduce anxiety, help learners to develop their ideas, and build connections. Conversely, lack of community building negatively inhibits the development of a sense of belonging and may impact upon retention. Thomas et al. (2014) acknowledge that fostering a sense of belonging and trying to encourage learners to become part of the community and its interactions is a challenging task for tutors. This study engages with that need.

\section{Research}

We now introduce our work into online sense of belonging and outline our findings to date. We are a small action-research team comprising three tutors engaged in promoting and facilitating online learning and an experienced visiting researcher. 
Our primary research question has been the following:

- What are our onlinelearners' accounts of their sense of belonging within their current postgraduate studies?

Supplementary questions include:

- How do these online learners define sense of belonging? What does the term mean to them?

- Is having this sense of belonging relevant for these online learners or not?

- According to these learners, what does, or does not, promote for them a sense of belonging at the course and institution levels?

- How important are peers in promoting and sustaining a sense of belonging?

- What role does the tutor play in promoting a sense of belonging for these learners?

We desire to understand in what ways, if any, online learners find it relevant to have a sense of belongingand why. Also, as tutors, we want to know what helps to promote a learner's sense of belonging and what role tutors and peers might play in developing and maintaining it.

\section{Data Collection}

Our small-scale exploratory study was undertaken at a small niche university in Scotland, whose 5,000 enrolled learners take predominantly professional programmes at undergraduate and postgraduate levels in Health Sciences, Arts, Social Sciences, and Management. The institution has a developing strategy promoting online provision that uses a collaborative approach for all modules. Learners from three online modules were asked to assist. These modules have been run online for several years to enable worldwide recruitment and engagement of employed learners; they have consistently received significantly positive learner feedback.

We approached learners $(\mathrm{N}=50)$ studying two compulsory modules part-time in the fully online MSc in Professional and Higher Education programme. One is a first programme experience, while the second follows in the second or third year of study. Both embody a strong collaborative, community-based approach, with learners being introduced to resource materials through a variety of onlinejournals, videos, and narrated PowerPoint presentations. Online discussions are core activities. Learners are introduced at the outset to their roles and responsibilities within the online community. They are expected to participate weekly in structured individual and group activities. In regular synchronous sessions, learners have the opportunity to meet with others in breakout rooms and to develop questions to be posed to the community. Synchronous drop-in sessions provide space for learners to discuss their studies with either the module tutors or the programme leaders.

Another group of learners $(\mathrm{N}=10)$ was also approached. They were enrolled in the part-time online MSc in Dispute Resolution programme and were studying a compulsory module, Fundamentals of Dispute 
Resolution. Most were busy professionals employed in complaint handling. This module was the first to be followed and included programme induction activities. It followed a structured collaborative approach to learning akin to that described above and clustered around five core learning topics. Learners were expected to participate in weekly discussion board activities, which contributed to the assessment in four of the five topics. Learners were paired for two activities and were expected to use Skype or a phone to discuss the task at hand. Two synchronous sessions were held, with varying success. One-to-one sessions with the tutor via phone or Skype were offered at two points within the module.

With ethical approval from Queen Margaret University, Edinburgh, volunteers from the three selected modules were recruited through a strategy of announcements and discussion postings in the university's virtual learning environment. They were initially invited to complete an anonymous online survey comprising eight open-ended questions. The survey tool had been piloted with two learners who had previously completed modules in the MSc in Professional and Higher Education programme. Their feedback was valuable in fine-tuning the tool, which asked learners to concentrate upon their current module, to explain what sense of belonging meant to them, and to provide concrete examples of incidents that had had a positive or negative effect on their sense of belonging. Other questions explored the impact of peers, as well as of the tutor and the family, on sense of belonging.

The data provided by 12 survey respondents (18.5\% of the cohort) were collated by the visiting researcher. At the end of the survey, respondents were invited to undertake a 45-minute telephone interview. Two learners volunteered. A further invitation was sent to all other respondents, but no responses were received. This small number of responses is something that should be considered in any follow-up study.

Semi-structured telephone interviews were conducted by the visiting researcher, concentrating upon the interviewees' experiences in their current module. Files of the interview transcriptions were shared with interviewees for editing to ensure their agreement with the record. No changes ensued or were requested.

In our research, we strove for trustworthiness and authenticity. Contacts with volunteer participants were undertaken by a senior academic (the visiting researcher) with no connection to the learning experiences. The analysis of the returned questionnaire and interviews was also undertaken independently. The arrangements for confidentiality in the survey and subsequent interviews precluded identification of the modules that individual volunteers were studying.

Although our participant numbers were small, much of the emerging open-ended data implies frankness and honesty, was informative to the teachers, and is expected to be of wider interest.

\section{Data Analysis}

Thematic analysis (Creswell, 2014) was used to analyse both the qualitative responses received from the survey and the telephone semi-structured interviews (which added considerable depth). First, the responses to each question in the survey were grouped under meaningful subheadings. The analysis of returned questionnaires followed this approach:

- Assemble quotations under the questionnaire questions as main headings. 
- Subdivide quotations under headings that describe a word or concept in the quotes.

- Rearrange quotations under subheadings to relate to something of a story line.

- Use all the submitted text except the odd conjunction; otherwise omit nothing.

- Add nothing other than the descriptions of what subheadings entail; refrain from editing.

- Omit all identification numbers to ensure confidentiality.

For example, when asked if a sense of belonging was important to them as online learners, all respondents replied "Yes" and gave short rationales, which were then themed into the following subheadings: Benefit, Motivation, Connections, Supportive Interactions, and Need (which was about isolation and socialisation).

The telephone interviews were structured around questions based upon significant questionnaire responses. Responses were transcribed by the visiting researcher, who eliminated any repetitions, pauses, and muttering. Sections that might cause identification of the interviewee were redacted. Otherwise, no editing or selection was done.

The materials were reordered in relation to the emerging survey themes. The reordered transcripts were reviewed by the research team, who sought deeper meaning from the points raised in questionnaire responses. The semi-structured interview transcripts were then thematically analysed using a concept mapping approach (Kane \& Trochim, 2007) to identify the main concepts and sub-concepts. The subheadings from the survey were also concept mapped, and each set of initial concepts from the content elicited from the participant group was reviewed. A member of the programme team independent of the research undertook an independent mapping exercise to ensure the veracity of the three main initial concepts engagement, culture of learning, and support.

\section{Findings}

This research aimed to establish the origins, nature, and importance (or not) of a sense of belonging in the lived experience of online learning for certain postgraduate learners; what a sense of belonging meant to them; and which facets of the online learning experience were important to them in relation to sense of belonging.

The three main concepts emerging from the analysis of the questionnaires and semi-structured interviews are explored in the following sections, which include quotations from the survey responses and the interviews to ensure that learners' voices are heard. Crucially, all 12 respondents identified sense of belonging as important to them as online learners, as it could prevent isolation. One opined that it is essential for all learners, but more so for online learners, as they are remote and lack the regular meeting and socialisation of face-to-face delivery of learning. Respondents variously believed that a sense of belonging was a "feeling" of being part of a community, of belonging to a group of learners with a common goal, and of engaging with learning materials and other learners and tutors. 


\section{Engagement}

The concept of engagement featured in all responses within the survey and related to tutors and learners. Tutors were identified by some respondents as being pivotal to the development of learners' sense of belonging, with comments such as "tutors are the glue that bring it together." Specifically, respondents identified that the tutor's attitude and approach set the tone of the learning and the development of a sense of belonging by safeguarding the way learners behave through their friendliness, helpfulness, and enthusiasm, and by maintaining contact via discussions, announcements, and posts-all of which assisted in reinforcing a sense of belonging. Learners noted that tutors "had an impact on [their] sense of belonging by their openness and honesty" and that "by having a continual presence during online discussions and through the short videos...the tutors [are brought] right into my dining room!" One respondent specifically identified "the glue" as "the quality of the learning materials put together, the guided reading and many references, the encouragement and feedback. These are all intrinsic to feeling valued and supported and key in sustaining that overall sense of belonging."

Learner involvement and interaction was also highlighted as critical in maintaining a sense of belonging during the modules. This related mostly to the nature and depth of posts in the discussions, along with engagement with group work and activities. The introductory element of getting to know each other, as well as and prior to the developing interactions, was indicated as crucial: "The encouragement to share information about ourselves and our histories made me feel a sense of belonging and connectedness with other students"; "Through the discussion boards I get to know my peers and therefore feel a sense of belonging."

This idea was followed up in the interviews, wherein one respondent noted that "although you have never met them physically face to face. . it's amazing how you do get glimpses of people's personality from the sort of online interactions going on."

One interviewee suggested that some of the sense of belonging might be related to personal situation or context. This learner had been in a new job, was very isolated, and felt something had been "lost"; "That's when I really had the sense of belonging to a community online because I was physically so far away from everyone."

The use of group work and other paired activities was seen as a strong method of developing a sense of belonging, as learners were actively expected to collaborate with their co-learners to achieve end goals by a certain allocated time: "In group tasks we have been able to help each other out by taking on a bit extra here and there at various times"; "It's good to have someone to help and make you feel like you are together in a class."

The online discussions were identified by learners as another strength in developing a sense of belonging, with the importance of engaging in discussion, by offering feedback and contrasting views, highlighted as a positive way of supporting and encouraging each other: "I think the discussion boards were helpful in this respect (peer impact on sense of belonging) as it meant getting to know other students and acted as an ongoing conversation albeit not in real time." 
A complementary perspective was presented by one respondent who identified that missing online discussions had a negative impact on their sense of belonging: "If I feel disconnected it is invariably because my own work commitments have taken over and I have missed out on online discussion or distanced myself from the learning materials."

Interview responses concurred with these views, with more in-depth detail obtained from interviewees who noted that interactions associated with group activities were mainly initiated by the learners-because they had a shared purpose of wishing to do well and supporting each other in achieving that. Most discussion interactions were triggered by a tutor-designed thread, which was usually based on the activities in the module; however, subsequent learner engagement in these was individually driven.

\section{Culture of Learning}

The concept of a culture of learning emerged from commentary around incidents that impacted positively or negatively on learners' sense of belonging. The culture of the modules within the learning group affected learners' sense of belonging through the module structure, the tutors' behaviours, and the materials, along with consequent learner behaviours.

Issues related to positive development of a sense of belonging that emerged were the notion of sharing and also the aspect of online learning materials being accessible over time. For this particular set of learners, responses suggested that the sharing of challenges and anxieties as learners in discussion groups had a powerful impact on the development of sense of belonging, as illustrated in the following quotation:

When I first started the module, I was very stressed and anxious with the platform and the material and everything else. Despite the tutor's reassurance, it was only when I saw similar concerns being posted on the discussion hub that I felt slightly at ease that I was not alone in trying to figure out how everything works. It was quite a relief!!!

In offering their advice to other learners regarding developing a sense of belonging, respondents identified the online discussions as valuable, promoting the flexibility of thinking seen as intrinsic to transformative learning. Similarly, they reported that they had encouraged peers to be unafraid to challenge, question, and/ or disagree with peers, as doing so enhances the quality of discussions.

The use of the discussion board by tutors was also seen as promoting a sense of belonging, with learners suggesting that when tutors posted their own views of things relating to the subject matter, learners got to know tutors a little more "as humans." Learners also valued feedback and the feeling that the tutor was regularly "present" online. They advised "Be visible, be present and offer as much live feedback as possible.. It makes the connection more obvious"; "Put up your own views on things relating to the subject matter, so they [learners] can get to knowyou a bit more."

A noteworthy strength of the online modules was the continued availability of materials and discussions as the modules progressed, with one learner commenting,

Due to illness I was unable to complete unit 3 of work for the module in first year. I have only been able to reconnect with this after everyone else has completed and submitted their assessment. 
Despite this, being able to access the online discussions/thoughts still makes me feel part of this learning community.

Conversely, some aspects of online learning were found to detract from learners' sense of belonging. Specifically, some aspects of feedback, or lack thereof, seemed to resonate with the learners as having a negative impact. The notion of off-putting behaviour emerged; specific behaviours were seen as detrimental to a sense of belonging, such as not posting any comment on discussion threads; cancelling an arranged session to do some group work, and then not responding to efforts to rearrange the time; and misunderstandings of feedback during group work activities or in discussions.

Providing feedback on a peers' work is therefore a minefield in terms of how it will be interpreted. This occurred once when a student wrote that another student's work "suffered from spelling errors." Cue a really long thread about hurt, support, female solidarity, etc, etc.

The whole aspect of online group work seems to present pitfalls in terms of maintaining a sense of belonging: "If people write back and/ or offer feedback in a derogatory manner that lessens your sense of belonging."

However, the sense of achievement of a common overall goal by the group seemed to outweigh negative aspects of feedback:

During the group tasks things got a bit fraught where there were some occasional misunderstandings of the online feedback-but these queries were swiftly cleared up as the overall goal of completing the task took over, and by the end the sense of achievement had obliterated any temporary individual "difference of opinion."

\section{Support}

The third concept derived from the research was the notion of support, which was interwoven throughout the questionnaire responses. Aspects of support that were highlighted were the sharing of issues, offering advice and views on aspects of the module, module design, and family support.

Support and constructive connections were specifically described as peer group interaction assisting in resolvinglearning difficulties. When learners felt stressed, help and feedback from peers and tutors through the discussion groups proved valuable, as it helped them to construct and develop their learning, offering the opportunity to "check things out." Informal connection with learners in the module seemed distinctly supportive, giving learners the feeling that they were not alone in their struggles: "I was happy to see that other people were having similar difficulties, which in turn significantly reduced my anxiety over the summative submission"; "In forum discussion, peers provide much needed guidance when there is some issue that needs to be further explored or revisited but also to bounce ideas off."

Results from the survey clearly showed that the module design and tutor/peer engagement were critical in developing a sense of belonging. The provision of engagement activities from the very start seems important in developing a connection between tutor and learners, as well as between learners. Use of multiple-media aspects, rather than a full-text base, also featured positively in maintaining a sense of belonging. Short 
videos to be viewed, activities of differing sorts-not always group work and all forms of interaction beyond the virtual learning environment were seen as important. Group work and (conversely) nonengagement feature in the findings, with differing views of mandatory assessed work to promote engagement (the "stick approach") versus engagement in working for learning (the "carrot approach"). Similar views were expressed in respect of discussion board contributions: "Assessed discussion boards means everyone contributes and the conversation continues"; "Try to apply peer pressure in group projects, by having some sort of (recorded) tele-meetings (or logbooks or other) in order for all members of the group to equally participate and contribute."

Online collaborative sessions were valued highly for their "live" interaction and theimmediacy of discussion and commentary. Tutor posts via e-mail or the virtual learning environment, with updates, interesting articles, and similar inputs, were also valued as supportive, as learners felt the tutor was always there for them.

Equally valued were the creation and use by learners of private locations or spaces within which they could engage in discussions and provide assistance in elucidating content and requirements of which formative activity they wished their tutors to be unaware.

This significance of interactive and engaging activities was summed up by an interviewee comparing their current online learning experience with a previous one:

I had started an online TEFL [Teaching English as a Foreign Language] course but this simply consisted of reading text online and completing activities related to it. There were no audiovisual materials, no interaction with other people on the course... I found it hard to motivate myself to complete the course and gave up.

\section{Discussion}

Our pilot-study learners believed a strong sense of belonging was of importance for them, and particularly so as online learners, being not physically present at the institution. Such findings concur with those of Thomas et al. (2014). Sense of belonging also appeared to increase learners' likelihood to remain on their course, to complete their studies and provided them with an extra level of resilience, especially in times of stress or anxiety, or simply when there were issues such as limited access to expected resources or inaccurate, limited, or late feedback.

Our work also identified the complexity of the "SoB feeling"-that is, the sense of belonging, being multifaceted, fluctuating, and ephemeral in nature, and differing for learners according to the context of their studies, individual needs, and self-efficacy. As Carruthers Thomas (2019) suggests, sense of belonging is "negotiated in the momentary, the imaginary and the private" (p. 76). Nowhere was this more evident to the researchers than when one interviewee described two unfortunate experiences of lack of support and direction in the initial enrolment and academic mismanagement of assessment. Nevertheless, the tenor of that interview was of a distinctly positive course experience and a strong overall sense of belonging. 
Throughout the study, it emerged that interactions with tutors and peers and professional services are critical in the development and maintenance of an online sense of belonging. Learners want to be known by their tutors "as individuals rather than reference numbers" and to have a relationship with them. The tutor guides learners through their studies, signposting potential routes forward and thus helping them to feel that they matter and that their presence would be missed. Furthermore, learners with a strong sense of belonging were forgiving of problems if they had formed the impression of a well set-up course that generated a strong, inherent sense of belonging. This reiterates O'Keeffe's (2013) findings, in working with campus-based learners, that "the creation of a caring, supportive and welcoming environment within the university is critical in creating a sense of belonging” (p. 605).

While the importance of tutors in planning and facilitating structured activities contributing inter alia to the development of a strong sense of belonging was expected within our study, the substantial influence of peers was surprising. Comments such as "If I do not feel I know the other learners, I do not feel I belong" were common. There was a belief that having relationships with others following the same programme and being part of a community helped with understanding within the programme and provided a better learning experience. One learner explained, "peers are the community that I am working within, and therefore all interaction with them provides the belonging." While this finding links with Koole and Parchoma's (2013) description of belonging in online learning communities as an iterative process of dialogue and exchange with other members, it is contrary to the finding of researchers in Australia that many online learners were expecting to be "lone wolves" working individually and in isolation from their peers (Brown, Hughes, Keppell, Hard, \& Smith, 2015).

The ethos of our online spaces clearly impacted on learners' sense of belonging. For instance, synchronous meetings, as identified by participants in Thomas et al.'s (2014) study, were particularly popular, fostering connections with others. Online interactions were also seen as a means of levelling the playing field by minimising differences in culture, gender, and discipline, which might otherwise inhibit some of the group activities and learners' sense of belonging. As one learner noted, 'It was possible to feel a sense of belonging and community in both the larger and smaller group, regardless of culture, professional status, or professional backgrounds."

The level, timeliness, and quality of support were considered important in promoting sense of belonging. Brief interactions and guidance from tutors and peers helped learners feel more comfortable in the online context and more confident in their abilities. Learners talked about tutors being "a continual presence during online discussions and through short videos," which helped make the alien environment less impersonal and more a place to go for help and guidance.

\section{Recommendations for Tutors}

Our final supplementary research question addressed the role of the tutor in promoting an online learner's sense of belonging. We learned that a supportive, facilitative tutor can effectively help online learners to develop and maintain a sense of belonging throughout their studies. Others have certainly noted the importance of a caring, enthusiastic tutor who can be trusted (Hoffman et al., 2002-2003; Strayhorn, 2012); this was particularly emphasised by our learners - at a distance and without the traditional face-to-face reassurance of tutors. Early introductions to the programme team through short videos and synchronous 
sessions seem to be of great potential in helping build bridges and develop trusting, nurturing tutorial relationships.

The role of the community and peers in developing sense of belonging was a key feature for our participants. Early opportunities in online discussions for learners to introduce themselves and share experiences, their reasons for undertaking studies, and their long-term goals, assisted in group formation and the development of peer support networks. Collaborative activities in which learners work together, for example, in the development of an artefact, linked closely to assessment, can develop peer relationships and promote feelings of being accepted, mattering, and being valued.

\section{Limitations of the Study}

This article has reported the first stages of an ongoing action-research study into sense of belonging for online learners. We accept that our work involved a small sample who developed a sense of belonging in particular circumstances. However, such early findings have already helped us to gain and provide sufficient insights into sense of belonging in the online educational space to inform further research studies during a group project set up by the UK Quality Assurance Agency in Scotland to produce a tool kit for online tutors on promoting sense of belonging (Quality Assurance Agency, 2019).

Our combined data-collection tools of open-ended survey questions followed by interviews have worked well in terms of the richness of the data generated, leading to the identification of our three themes. The visiting researcher noted the extent and quality of the additional evidence that was gathered during the 45minute interviews, providing considerable depth of insight into some matters already identified in more general terms in the questionnaire responses. In the future, we will refine our tools to ensure that more space is available for learners to articulate their understandings of sense of belonging.

\section{Conclusions}

Many publications have emphasised the importance of sense of belonging for onlinelearners and especially those who are considered to be at risk of course non-completion (O'Keeffe, 2013). However, little seemed previously known in detail about a sense of belonging on the part of online learners who are engaging in collaborative, community-based learning; and even less was known about how institutions and tutors can promote it. Online learners in a new and potentially alienating environment, remote from the physical campus and separated physically from their peers, seem to be especially in need of a sense of belonging. In their testimonies, our learners valued tutors who created a sense of belonging in their study experience; we found that for these online learners, this tutor effort created more resilience and more connection to their studies, their peers, and their tutors, and it assisted them in remaining in the course and, indeed, flourishing. 
Peacock, Cowan, Irvine, and Williams

\section{Acknowledgements}

The authors are grateful to the reviewers for their collegial efforts. We would particularly like to thank one reviewer who devoted considerable efforts to compiling a thoughtful list of perceptive, constructive, and scholarly comments on the submitted draft. These have contributed significant enhancement to the final version. 


\section{An Exploration Into the Importance of a Sense of Belonging for Online Learners \\ Peacock, Cowan, Irvine, and Williams \\ References}

Allen, E., \& Seaman, J . (2013). Changing course: Ten years of tracking online education in the United States. Retrieved from http:// www.onlinelearningsurvey.com/reports/changingcourse.pdf

Baumeister, R. F., \& Leary, M. (1995). The need to belong: Desire for interpersonal attachments as a fundamental human motivation. Psychological Bulletin, 117, 497-529. https:// doi.org/ 10.1037/ 0033-2909.117.3.497

Baxter, J . (2012). Who am I and what keeps me going? Profiling the distance learning student in higher education. The International Review of Research in Open and Distributed Learning, 13(4), 108129. https:// doi.org/ 10.19173/irrodl.v13i4.1283

Brown, M., Hughes, H., Keppell, M., Hard, N., \& Smith. L. (2015). Stories from students in their first semester of distance learning. The International Review of Research in Open and Distributed Learning, 16(4). https:// doi.org/ 10.19173/irrodl.v16i4.1647

Carr, S. (2000). As distance education comes of age, the challenge is keeping the students. The Chronicle of Higher Education, 46(23), 39-41. Retrieved from https:// www.chronicle.com/ article/ AsDistance-Education-Comes-of/ 14334

Carruthers Thomas, K. (2019). Rethinking student belonging in higher education: From Bourdieu to borderlands. London, UK: Routledge.

Creswell, J . (2014). Research design: Qualitative, quantitative, and mixed methods approaches (4th ed.). London, UK: Sage Publications.

Garrison, D. R., (2011). E-learning in the 21st century: A framework for research and practice (2nd ed.). New York, NY: Routledge.

Goodenow, C. (1993). Classroom belonging among early adolescent students: Relationships to motivation and achievement. The J ournal of Early Adolescence, 13(1), 21-43, https:// doi.org/ 10.1177/ 0272431693013001002

Hagerty, B., Williams, R., Coyne, J ., \& Early, M. (1996). Sense of belonging and indicators of social and psychological functioning. Archives of Psychiatric Nursing, 10, 235-244. https:// doi.org/ 10.1016/ S0883-9417(96)80029-X

Hausmann, L. R. M, Ye, F., Schofield, J ., \&Woods, R. (2007). Sense of belonging and persistence in white and African American first-year students. Research in Higher Education, 50(7), 649-669. https:// doi.org/ 10.1007/ s11162-009-9137-8

Hoffman, M., Richmond, J ., Morrow, J ., \& Salomone, K. (2002-2003). Investigating “sense of belonging” in first-year college students. J ournal of College Student Retention: Research, Theory \& Practice, 4(3), 227-256. https:// doi.org/ 10.2190/ DRYC-CXQ9-J Q8V-HT4V 
Hurtado, S, \& Carter, D. (1997). Effects of college transition and perceptions of the campus racial climate on Latino college students' sense of belonging. Sociology of Education, 70(4), 324-345.

Illeris, K. (2014). Transformative Learning and Identity.J ournal of Transformative Education, 12(2), 148- 163. https:// doi.org/ 10.1177/ 1541344614548423

Jackson, P., Cashmore, A., \& Scott, J . (2010). Sense of belonging: Background literature. Retrieved from https:// www2.le.ac.uk/ offices/ssds/projects/student-retention-project/ dissemination/ papersand-publications/Sense\%20of\%20Belonging\%20Lit\%20Review.docx

Jarvis, P. (2009). Learning to be a person in society. In K. Illeris (Ed.), Contemporary theories of learning: Learning theorists - In their own words (pp. 21-34). Routledge: London. https:// doi.org/ 10.4324/9780203870426

Johnson, D., Soldner, M., Leonard, J ., Alvarez, P., Inkelsa, K., Rowan-Kenyon, J ., \&Longerbeam, S. (2007). Examining sense of belonging among first-year undergraduates from different racial/ ethnic groups. J ournal of College Student Development, 48, 525-542. https:// doi.org/ 10.1353/csd.2007.0054

Kane, M. \& Trochim, W. M. (2007). Concept mapping for planning and evaluation. Baltimore, MD: Sage Publications.

Koole, M., \& Parchoma, G. (2013). The web of identity: A model of digital identity formation in networked learning environments. In S. Warburton \& S. Hatzipanagos (Eds.), Digital identity and social media (pp. 14-28). Hershey, PA: IGI Global. https:// doi.org/ 10.4018/978-1-4666-1915-9.ch002

Laurillard, D. (2012). Teaching as design science. Abingdon, UK: Routledge.

Locks, A., Hurtado, S., Bowman, N., \&Oseguera, L. (2008). Extending notions of campus climate and diversity to students' transition to college. The Review of Higher Education, 31(3), 257-285. https:// doi.org/ 10.1353/rhe.2008.0011

Matheson, R., \& Sutcliffe, M. (2018). Belonging and transition: An exploration of international business students' postgraduate experience. Innovations in Education and Teaching International, 55(5), 602-610. https:// doi.org/ 10.1080/ 14703297.2017.1279558

Meehan, C., \& Howells, K. (2018). What really matters to freshers? Evaluation of first year student experience of transition into university. J ournal of Further \& Higher Education, 42(7), 893-907. https:// doi.org/ 10.1080/0309877X.2017.1323194

O'Keeffe, P. (2013). A sense of belonging: Improving student retention. College StudentJ ournal, 47(4), 605-613. Retrieved fromhttps:// researchbank.rmit.edu.au/view/rmit:33763

O'Shea, S., Stone, C., \&Delahunty, J . (2015). “I 'feel' like I am at university even though I am online.” Exploring how students narrate their engagement with higher education institutions in an online 
learning environment. Distance Education, 36(1), 41-58.

https:// doi.org/ 10.1080/01587919.2015.1019970

Peacock, S., \& Cowan, J . (2017). Towards online student-directed communities of inquiry. J ournal of Further and Higher Education, 42(5), 678-693.

https:// doi.org/ 10.1080/0309877X.2017.1302569

Peacock, S., \& Cowan, J . (2019). Promoting sense of belonging in online learning communities of inquiry in accredited courses. Online Learning, 23(2), 67-81. https:// doi.org/ 10.24059/olj.v23i2.1488

Quality Assurance Agency. (2019). Distance and sense of belonging. Retrieved https:// www.enhancementthemes.ac.uk/ current-enhancement-theme/student-engagement-anddemographics/distance-and-sense-of-belonging

Strayhorn, T. (2012). College students' sense of belonging: A key to educational success for all students. Oxon, UK: Routledge.

Thomas, L. (2012). Building student engagement and belonging in higher education at a time of change: A summary of findings and recommendations from the what works? Student retention \& success programme. London, UK: Paul Hamlyn Foundation. Retrieved from https:// www.phf.org.uk/ wp-content/ uploads/ 2014/ 10/What-Works-Summary-report.pdf

Thomas, L., Herbert, J ., \& Teras, M. (2014). A sense of belonging to enhance participation, success and retention in online programs. The International J ournal of the First Year in Higher Education, 5(2), 69-80. https:// doi.org/ 10.5204/intjfyhe.v5i2.233

Thomas, L., Herbert, J ., \& Teras, M. (2014). A sense of belonging to enhance, participation, success and retention in online programs. The International J ournal of the First Year in Higher Education, 5(2) 69-80. https:// doi.org/ 10.1177/00131644093444510

Vaccaro, A., Daly-Cano, M., \&Newman, B. M. (2015). A sense of belonging among college students with disabilities: An emergent theoretical model. J ournal of College Student Development, 56(7), 670686. https:// doi.org/ 10.1353/ csd.2015.0072

Vaccaro, A., \&Newman, B. (2017). A sense of belonging through the eyes of first-year LGBPQ students. J ournal of Student Affairs Research and Practice, 54(2), 1-13. https:// doi.10.1080/19496591.2016.1211533

Whittaker, A. (2015). Effects of team-based learning on self-regulated online learning. International J ournal of Nursing Education Scholarship, 12(1), 110. https:/ / doi.org/ 10.1515/ijnes-2014-0046 
An Exploration Into the Importance of a Sense of Belonging for Online Learners

Peacock, Cowan, Irvine, and Williams

Athabasca

University

(c) 